ISSN 0103-5150

Fisioter. Mov., Curitiba, v. 25, n. 1, p. 195-205, jan./mar. 2012 Licenciado sob uma Licença Creative Commons

\title{
Exercício resistido no cardiopata: revisão sistemática
}

\author{
Resistance exercise in heart disease: \\ systematic review
}

\section{Ana Clara Campagnolo Real Gonçalves ${ }^{[a]}$, Carlos Marcelo Pastre ${ }^{[b]}$, José Carlos Silva Camargo Filho $^{[\mathrm{cl}}$, Luiz Carlos Marques Vanderlei ${ }^{[\mathrm{d}]}$}

[a] Mestre em Fisioterapia pela Universidade Estadual Paulista (UNESP), Departamento de Fisioterapia, Presidente Prudente, SP - Brasil, e-mail: clara_camp@ig.com.br

[b] Doutor em Ciências da Saúde, Universidade Estadual Paulista (UNESP), Departamento de Fisioterapia, Presidente Prudente, SP - Brasil, e-mail: pastre@fct.unesp.br

[c] Doutor em Cirurgia Experimental, Universidade Estadual Paulista (UNESP), Departamento de Fisioterapia, Presidente Prudente, SP - Brasil, e-mail: camargo@fct.unesp.br

[d] Doutor em Odontologia - área de concentração: Farmacologia, Universidade Estadual Paulista (UNESP), Departamento de Fisioterapia, Presidente Prudente, SP - Brasil, e-mail: lcmvanderlei@fct.unesp.br

\section{Resumo}

Introdução: A perda de massa muscular secundária à idade e à inatividade física é clinicamente relevante na população cardíaca; contudo, a prescrição do exercício resistido dinâmico para esses pacientes apresenta-se inconclusiva na literatura. Objetivos: Reunir informações e apresentar as principais diretrizes relacionadas à prescrição de exercícios resistidos em cardiopatas. Materiais e métodos: Foi realizada busca sistemática de literatura, a partir das bases de dados LILACS, SciELO e MEDLINE, utilizando os seguintes descritores na língua portuguesa: força muscular, exercício isométrico, esforço físico, cardiopatia e coronariopatia, e seus correspondentes na língua inglesa (muscle strength, isometric exercise, physical effort, heart disease e artery coronary disease), os quais foram pesquisados separadamente e em cruzamentos, sendo considerados para esta revisão apenas artigos publicados entre 2005 e 2010. Resultados e discussão: De um total de 806 artigos foram selecionados 22 para integrar a revisão, sendo 14 estudos classificados como artigos originais, 2 artigos de atualização da literatura e 6 artigos de revisão, além do capítulo 8 do livro intitulado Diretrizes do ACSM (American College of Sports Medicine) para os testes de esforço e sua prescrição, publicado em 2007. Conclusão: 0 exercício resistido, independente da 
variada metodologia utilizada na prescrição dos componentes específicos do treinamento, mostrou-se eficiente para aumentar a força muscular de membros superiores e inferiores em cardiopatas e sua aplicação pode ser considerada segura para esses pacientes, desde que prescrito corretamente.

Palavras-chave: Força muscular. Exercício isométrico. Esforço físico. Cardiopatia. Coronariopatia.

\begin{abstract}
Introduction: Loss of muscle mass secondary to age and physical inactivity is clinically relevant in cardiac population, however dynamic resistance exercise prescription to these patients presents inconclusive in literature. objectives: Collecting information and presenting the main guidelines related to resistance exercise prescribed in cardiac patients. Materials and methods: Systematic search was performed, using the data bases LILACS, SCIELO and MEDLINE. The descriptors used were: muscle strength, isometric exercise, physical effort, heart disease and artery coronary disease, which were surveyed separately and combined, considering for this review articles that were published between 2005 and 2010. Results and discussion: From a total of 806 articles, 22 were selected to compose the review, 14 original articles, 2 dates literature articles and 6 review articles, beyond the chapter 8 of the book ACSM's guidelines (American College of Sports Medicine) for exercise testing \& prescription, published in 2007. Conclusion: Resistance exercise, regardless of the varied methods used to prescribe the specific components of training, has proved effective for increasing muscle strength of upper and lower limbs in patients with and its application can be considered safe for these patients, provided that prescribed correctly.
\end{abstract}

Keywords: Muscle strength. Isometric exercise. Physical effort. Heart disease. Artery coronary disease.

\title{
Introdução
}

Exercícios físicos como terapia associada à terapia medicamentosa são largamente empregados em pacientes cardiopatas. Na literatura, encontram-se bem descritos os benefícios do treinamento físico aeróbico, seus riscos e suas limitações. Nesse âmbito, destaca-se a própria especificidade de estímulos relacionada à endurance e não ao aumento de força muscular (1). Por isso, a prescrição de exercícios resistidos associados ao treinamento físico aeróbico, anteriormente contraindicado para cardiopatas, tem sido recomendada visando a uma intervenção mais abrangente (2-4).

A perda de massa muscular secundária à idade e à inatividade física é clinicamente relevante na população cardíaca, pois associada a menores taxas metabólicas de repouso favorece o ganho de massa gorda e a diminuição do desempenho funcional. Somado a isso, esses pacientes apresentam alta prevalência de fatores de risco cardiovasculares, baixo nível de independência funcional e maiores índices de mortalidade. Além disso, reduções da força e da resistência muscular contribuem para aumento do risco de lesões por quedas e diminuição das aptidões físicas para atividades diárias (2-5).
Assim, sugere-se o aumento de massa muscular em cardiopatas, por meio de exercícios resistidos, a fim de promover: otimização da resposta do condicionamento aeróbico, aumento da densidade mineral óssea, aprimoramento do tecido conjuntivo, aumento ou manutenção do peso corporal magro, redução do risco de osteoporose e diabetes e controle da hipertensão arterial $(2,3,5)$.

Contudo, a literatura parece não apresentar consenso quanto às características da prescrição do exercício resistido, classificadas como seguras aos cardiopatas visando ao ganho de força, como valores da carga de intensidade e volume de trabalho, períodos de recuperação, frequência, duração e sobrecarga do exercício.

Portanto, o objetivo desta revisão é reunir informações e apresentar as principais diretrizes relacionadas à prescrição de exercícios resistidos em cardiopatas.

\section{Materiais e métodos}

A busca científica foi realizada nos meses de abril e maio de 2010, por meio de pesquisas nas bases de 
dados online MEDLINE (Medical Literature, Analysis and Retrieval System Online), SciELO (Scientific Eletronic Library Online) e LILACS (Literatura Latino-Americana e do Caribe de Informação em Ciência da Saúde).

\section{Critérios de inclusão}

Para as buscas nessas bases de dados, as palavras-chave foram selecionadas a partir dos Descritores em Ciências da Saúde (DeCS) e Medical Subject Headings (MeSH), sendo utilizadas as seguintes palavras-chave: muscle strength, isometric exercise, physical effort, weightlifting, artery coronary disease e heart disease. Nas bases de dados LILACS e SciELO foram também utilizados os termos em português: força muscular, exercício isométrico, esforço físico, levantamento de pesos, coronariopatia e cardiopatia.

A pesquisa de artigos na base de dados MEDLINE foi realizada a partir dos cruzamentos dos unitermos: muscle strength, isometric exercise, phyisical effort, weightliftin com artery coronary disease ou heart disease. Resultando, com a palavra-chave "artery coronary disease", 138 artigos; e com "heart disease" 295 artigos.

Já nas bases de dados LILACS e SciELO foram utilizadas isoladamente todas as palavras-chave na língua inglesa e portuguesa, e também em cruzamentos, como realizado para a base de dados MEDLINE. Na base de dados SciELO foram encontrados 351 artigos ao se realizar a pesquisa com todos os unitermos de maneira isolada, mas não foi encontrado nenhum artigo ao se repetir a busca, por meio do cruzamento dos descritores. Em contrapartida, na base de dados LILACS, foram obtidos 23 artigos referentes ao tema dessa revisão somente quando se usou o cruzamento das palavras-chave com o descritor "cardiopatia" ou "heart disease".

Foram considerados para o presente estudo apenas artigos publicados entre o período de 2005 a 2010. A pesquisa realizada originou um total de 804 artigos, os quais inicialmente tiveram seus resumos analisados para identificação daqueles que atendessem aos critérios de inclusão desta revisão. Os textos completos dos artigos potencialmente relevantes foram recuperados para avaliação final e suas listas de referências foram checadas para identificar estudos com potencial relevância não encontrados na busca eletrônica. Esse trabalho foi repetido até haver a convicção de que todas as referências obtidas já tivessem sido identificadas. Foram considerados para análise artigos publicados nas línguas inglesa e portuguesa.

Em função da importância e do valor científico das recomendações do American College of Sports Medicine (ACSM) para a prescrição de exercícios e levando em consideração que sua última publicação de diretrizes para prescrição de exercício em pacientes cardiopatas foi no ano 2000, foi incluído nesta revisão o capítulo 8 (Modificações na prescrição de exercício para pacientes cardíacos) do livro intitulado Diretrizes do ACSM para os testes de esforço e sua prescrição, publicado em 2007.

Tipo de estudo

Foram incluídos nesta revisão apenas artigos que abordassem pacientes portadores de cardiopatia, sendo selecionados ensaios clínicos randomizados controlados e não controlados e estudos de revisão baseados em treinamento resistido, associado ou não ao treinamento aeróbico. Foram analisados os componentes da prescrição do exercício e a sua influência sobre os sistemas cardiovascular, musculoesquelético e a qualidade de vida.

Dos 806 artigos iniciais foram selecionados 22 para integrar a revisão, sendo 14 estudos classificados como artigos originais, 2 artigos de atualização da literatura e 6 artigos de revisão, além do capítulo do livro anteriormente relacionado. 0 restante dos artigos encontrados não preencheu o principal critério para sua inclusão nesta revisão, ou seja, abordar exercício resistido em pacientes cardiopatas.

\section{Tipo de participantes e intervenção}

Foram analisados estudos com pacientes inseridos em programa de reabilitação cardiovascular supervisionado ou não supervisionado, independentemente da fase de intervenção (fase 1, fase 2 e fase 3 da reabilitação cardiovascular), que praticassem atividade física exclusivamente resistida ou associada ao exercício aeróbico. Graças ao limitado referencial bibliográfico referente ao tema proposto, optou-se ainda por não restringir a condição clínica da amostra de cada estudo e a modalidade do exercício resistido (dinâmico, isométrico, circuito, pesos 
livres, corda elástica ou aparelhos de musculação), associado ou não ao treinamento aeróbico.

Tipo de resultados relatados

Estudos que investigaram modificações e alterações, nas variáveis do sistema cardiovascular e muscular, relacionadas à prática de atividade resistida combinada ou não à atividade física aeróbica por pacientes que apresentam cardiopatia.

\section{Resultados e discussão}

A maioria de nossas atividades de vida diária inclui atividades como subir e descer escadas, levantar, empurrar, ou carregar algum objeto, as quais exigem força muscular na sua execução e, consequentemente, geram estresse sobre o sistema musculoesquelético. Adicionado a isso, muitos pacientes cardiopatas possuem redução da força física e/ ou da autoconfiança necessárias para a realização dessas atividades e, ao considerar as necessidades e os objetivos específicos dos indivíduos participantes de um programa de reabilitação cardíaca, o exercício resistido deve ser estimado $(1,5,6)$.

A atualização da literatura publicada por Adams et al. (6) relata que pacientes após evento cardíaco recebem orientações de médicos que implicam restrições excessivas e limitantes à prática de exercício resistido, o que aumenta a insegurança e a falta de motivação para o retorno às atividades rotineiras. Contudo, força e resistência muscular são importantes habilidades físicas que garantem ao indivíduo o retorno seguro e eficaz para executar as atividades de vida diária e profissionais. Assim, pacientes com evolução não complicada após infarto agudo do miocárdio (IAM) estão aptos para o retorno ao trabalho dentro de quatro semanas, e à prática do exercício de resistência na fase II da reabilitação cardíaca faz-se fundamental para essa readaptação.

Os artigos de revisão selecionados e o capítulo do livro da ACSM justificam a prática do treinamento resistido de maneira segura e eficaz para cardiopatas. Desde a primeira recomendação da American Heart Association (AHA) e do American College of Sports Medicine (ACSM) para o treinamento resistido, em 2000, essa modalidade de exercício tornou-se ainda mais aceita e utilizada em programas de exercícios para pessoas com e sem doenças cardiovasculares (DCV) (5). A Diretriz Brasileira de Reabilitação Cardíaca (3) e a ACSM (1) relatam que a força muscular é fundamental para a saúde, para a manutenção de boa capacidade funcional e para atingir qualidade de vida satisfatória.

Os benefícios potenciais do exercício resistido incluem não só melhora na saúde e controle de fatores de risco para doenças cardiovasculares como hipertensão arterial, dislipidemia, sensibilidade à insulina, melhor controle do peso, prevenção de deficiências e quedas e aumento da capacidade funcional (1-6).

A inclusão do treinamento de força em programas de reabilitação cardíaca produz efeitos favoráveis ao bem-estar geral do paciente, pois auxilia na melhora da força e resistência muscular, do metabolismo, da função cardiovascular, evidenciada a partir de aumento do consumo máximo de oxigênio e melhora do débito cardíaco e significante redução da percepção do esforço para atividades submáximas $(1,3,5,6)$.

Para esta revisão, dos 14 artigos originais selecionados quatro utilizaram o exercício resistido como intervenção isolada, somente um artigo avaliou um programa não supervisionado de treinamento resistido e os nove artigos restantes submeteram cardiopatas a um programa de treinamento resistido supervisionado com intervenção, associado ou comparado ao treinamento aeróbico (Tabela 1), e relataram benefícios no incremento de força e na melhora da capacidade funcional, sem a presença de complicações cardiovasculares ou lesões musculoesqueléticas durante o treinamento.

Estudos demonstram que pacientes com doença arterial coronária (DAC) e insuficiência cardíaca (IC) apresentam menor força muscular máxima de membros inferiores (MMII) quando comparados a indivíduos de mesma faixa etária e saudáveis (7-9).

Ghroubi et al. (7) avaliaram 30 voluntários entre cardiopatas e controles saudáveis, com o objetivo de investigar se a baixa capacidade aeróbica de pacientes com DAC está acompanhada pelo déficit de força muscular em MMII. Os autores observaram, por meio de teste de capacidade máxima, teste de caminhada de seis minutos e avaliação pelo dinamômetro isocinético, que os pacientes cardiopatas apresentaram redução da capacidade cardiorrespiratória e fadiga precoce nos músculos avaliados.

0 estudo de Okada et al. (8) verificou se a miopatia presente em pacientes portadores de IC está 


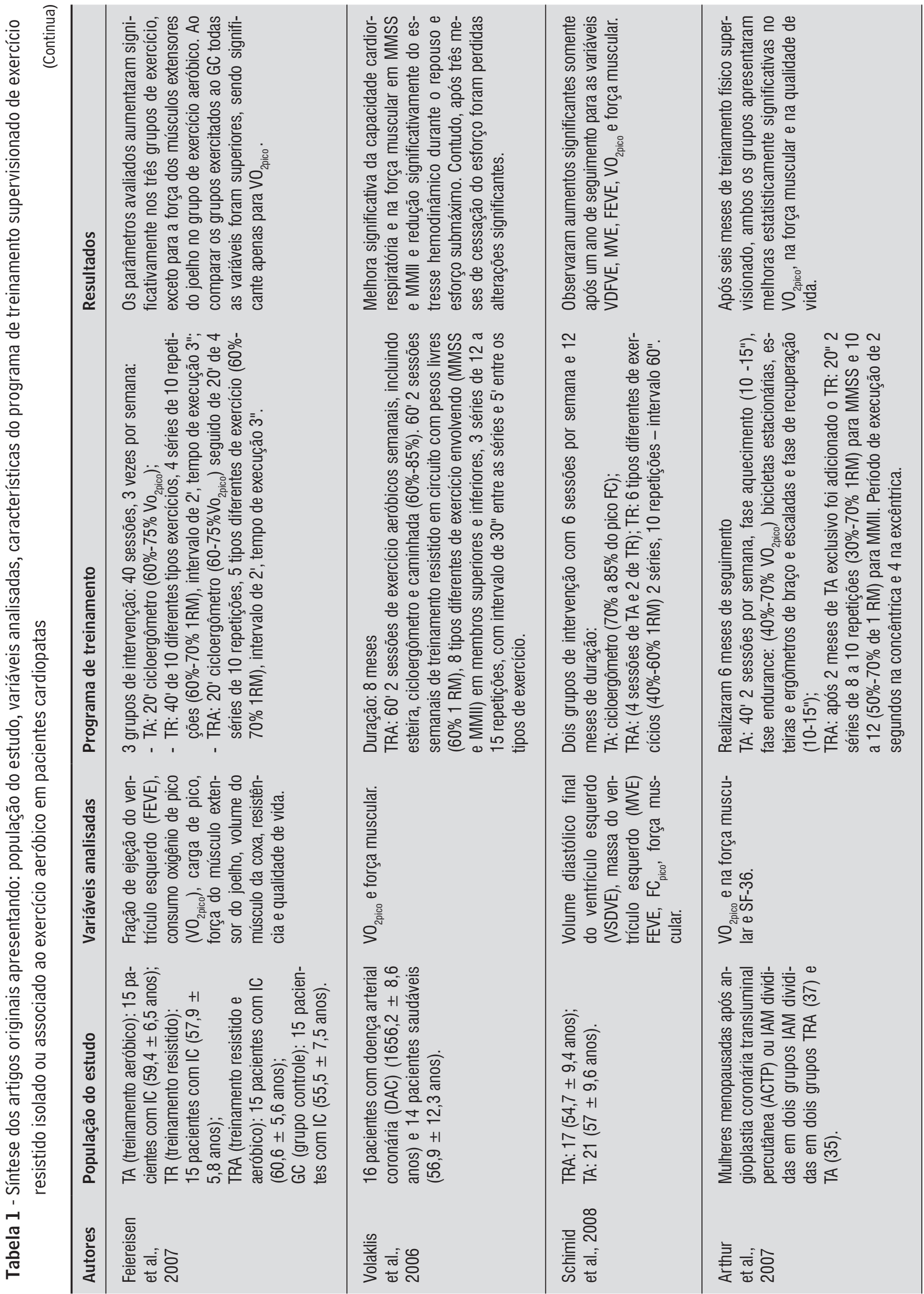




\begin{tabular}{|c|c|c|c|c|}
\hline & 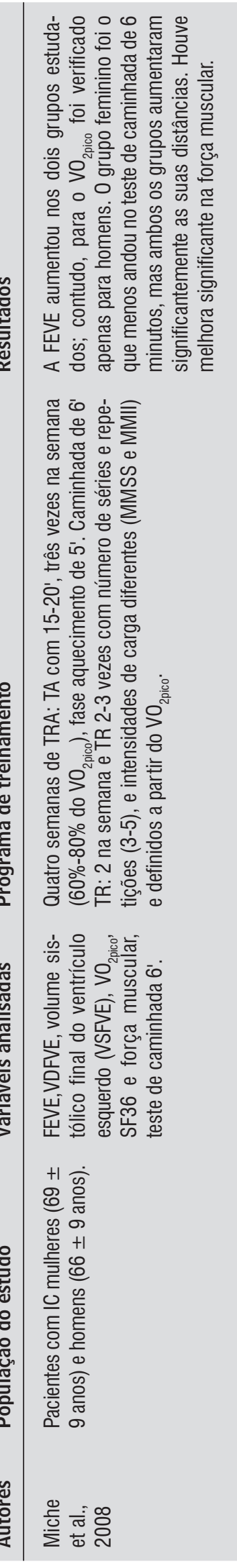 & 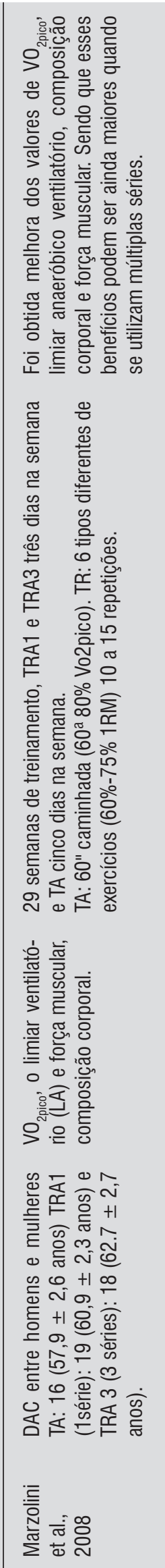 & 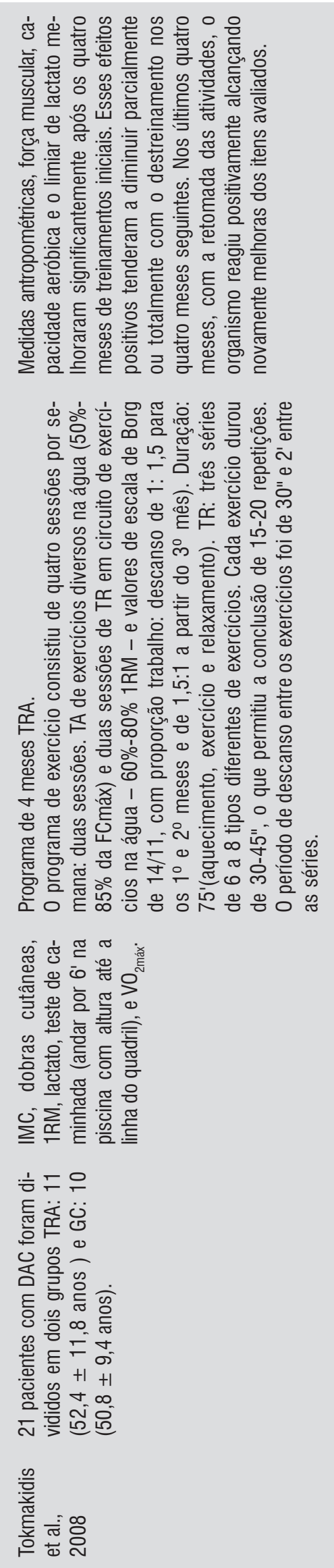 & 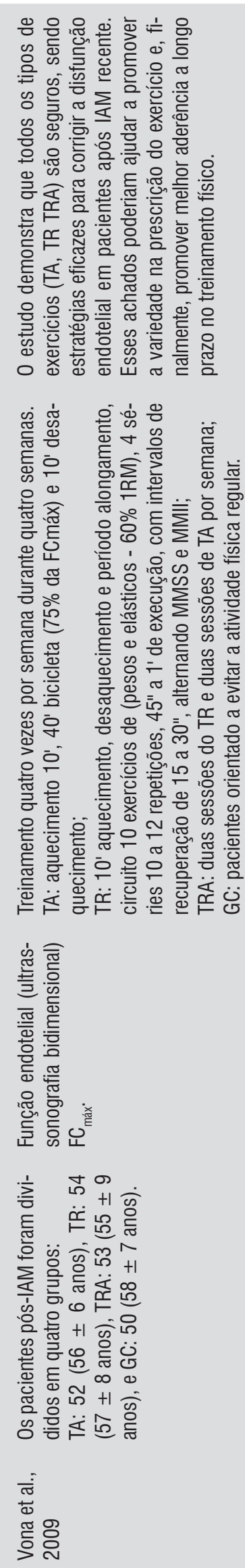 \\
\hline
\end{tabular}


associada a alterações funcionais das proteínas contráteis das fibras musculares. Foi observada redução da força musculoesquelética nesses indivíduos sem alterações específicas funcionais nos filamentos finos e para a miosina. Contudo, foi confirmado menor conteúdo dessas proteínas miofibrilares, o que pode justificar a fraqueza muscular desses pacientes.

Já o estudo publicado por Baum et al. (9) comparou a força muscular dos flexores de cotovelo e extensores de joelho de 638 cardiopatas, selecionados de programa de reabilitação cardiovascular, após 3 semanas de treinamento aeróbico e resistido, e 961 voluntários saudáveis, e observou declínio significativo da força muscular tanto em membros superiores (MMSS) quanto em MMII, em ambos os grupos, com o aumento da idade. Na análise intergrupos foi observado déficit de força significante apenas em MMII, sendo o subgrupo de pacientes cardiopatas, com histórico de inatividade física antes do evento cardíaco, o que apresentou maior fraqueza muscular.

Os estudos apontam que a fraqueza muscular em pacientes cardiopatas decorre da inatividade física e, particularmente nos pacientes com insuficiência cardíaca, é consequente da atrofia muscular, sendo essa uma das características que compõem o estado patológico do sistema musculoesquelético em consequência da cardiopatia (10). Esses estudos reforçam a importância da prática do exercício resistido, uma vez que a força muscular é uma aptidão física treinável e um fator importante para a execução das atividades de vida diária e profissionais $(1,11)$.

De acordo com a sistematização do presente estudo, não foram encontrados artigos, publicados no período de 2005 a 2010, que relatassem efeitos do exercício resistido sobre fatores de riscos cardiovasculares em portadores de cardiopatias, mas somente estudos que abordaram população jovem ou idosa que apresentou isoladamente fatores de risco, como hipertensão arterial, diabetes mellitus ou dislipidemias, os quais não preencheram o principal critério para sua inclusão nesta revisão.

Entre os artigos selecionados, medidas antropométricas foram avaliadas somente por dois ensaios clínicos, o primeiro de autoria de Tokmakidis et al. (12) e o segundo por Marzolini et al. (4). Nesse primeiro artigo, após quatro meses de treinamento aeróbico e resistido na água, seguido por avaliação após quatro meses de interrupção do treinamento e, por fim, após os quatro meses seguintes, no período de retreinamento, os autores observaram melhoras significativas nos valores de índice de massa corpórea (IMC) e das dobras cutâneas após o treinamento, sendo essas melhoras perdidas com a cessação do treinamento. Após quatro meses do retorno das atividades, constataram-se novamente as melhorias verificadas na fase inicial da intervenção.

Melhorias na composição corporal também foram observadas no artigo de autoria de Marzolini et al. (4), sendo avaliadas por meio de absorciometria com raios $\mathrm{x}$ de dupla energia (DEXA) antes e após treinamento aeróbico isolado (TA) e treinamento associado resistido e aeróbico com uma série (TRA1) e com três séries (TRA3). Observaram-se alterações positivas significativas no aumento na massa magra total nos grupos TRA1 e TRA3 e uma tendência para o aumento dessa variável no grupo TA. Contudo, não foram verificadas mudanças significativas em nenhum dos grupos estudados para as medidas de cintura e circunferência do quadril, ou índice de massa corporal.

Estudos publicados com a intervenção do treinamento resistido apresentam variada descrição dos componentes para sua prescrição. Fator que dificulta para agrupá-los, uma vez que em todos os artigos selecionados foram obtidos ganhos de força e capacidade funcional, sem oferecer risco cardiovascular ou de incidência de lesões musculoesqueléticas em suas populações. Dessa maneira, serão apresentados nove ensaios clínicos e seis revisões da literatura e um capítulo do livro do ACSM sobre esse tema.

De acordo com o capítulo do livro do ACSM (1), caracteriza-se o exercício especificamente como resistido quando realizado com carga entre $50 \%$ a $100 \%$ da carga máxima atingida no teste de repetição máxima (1RM). Para pacientes cardiopatas, recomendam-se de 8 a 10 tipos diferentes de exercícios, que envolvam os principais grupos musculares e uma série de 10 a 15 repetições, sempre respeitando os valores de 11 a 13 na escala de Borg. Além disso, o duplo-produto alcançado durante o treinamento não pode ser superior ao alcançado na atividade física aeróbica, prescrita de acordo com a estratificação de risco cardiovascular do paciente. Durante a execução dos exercícios o ritmo das repetições deverá ser controlado para que sejam lentos e a manobra de Valsalva deve ser evitada. Para iniciar a atividade sugerem-se halteres leves com carga entre $990 \mathrm{~g}$ a 2.270 g para membros superiores e 2.270 a 4.500 g para membros inferiores. 
0 estudo de Lamotte et al. (13) sugere que o treinamento resistido aos cardiopatas deve ser inciado a partir de 2 a 3 séries de 8 a 10 repetições com pesos correspondentes a $30 \%-40 \%$ de $1 \mathrm{RM}$, o que deve aumentar a autoconfiança dos pacientes ao treinamento, e que a sobrecarga seja aumentada de maneira progressiva e gradual, preferencialmente aumentando o percentual da intensidade de carga e o número de séries, em vez do número de repetições, até alcançar o máximo de $70 \%$ da força de contração voluntária máxima previamente avaliada.

Corroboram-se as recomendações da Diretriz Brasileira de Reabilitação Cardiovascular (3) e da revisão de autoria de Vicent and Vicent (5), as quais definem que a sobrecarga do esforço deve ser aplicada a partir da evolução do peso do componente estático, de maneira gradual e progressiva. Contudo, eles diferem na maneira pela qual deve ser realizado esse incremento: a diretriz sugere aumentar pelo percentual da carga máxima obtida no teste prévio e apresenta limite de intensidade entre $50 \%-60 \%$ RM, já a revisão sugere aumentos 1 a $3 \mathrm{~kg}$ por semana, controlados a partir dos valores limites determinados na escala de Borg, ou dos valores de duplo produto cardíaco.

Outro componente importante na prescrição do exercício resistido é a definição do número de séries, no estudo de Marzolini (4), em que, por meio da combinação de treinamento aeróbico associado a treinamento resistido com cargas progressivas, $60 \%$ a $75 \%$ do $\mathrm{VO}_{2}$ pico e $60 \%$ a $75 \%$ da $\mathrm{RM}$, objetivou-se avaliar se diferentes números de séries ( 1 x 3 ) implicariam melhores benefícios ao cardiopata. Nesse trabalho os autores relataram que os diferentes protocolos associando o exercício aeróbico ao resistido foram bem tolerados pelos voluntários, além disso, foram obtidas melhoras nos valores de $\mathrm{VO}_{2}$ pico, limiar anaeróbico ventilatório, força e endurance musculares e na composição corporal, quando se comparou ao grupo treinado exclusivamente por exercícios aeróbicos, sendo que esses ganhos podem ser ainda maiores quando se utilizam múltiplas séries.

Na associação do exercício resistido ao aeróbico, Volaklis et al. (14) obtiveram aumento em média de 28\% na força muscular de MMSS e MMII e melhora da aptidão cardiorrespiratória em coronariopatas, com a realização por oito meses de um programa de exercício supervisionado com frequência de quatro vezes por semana, sendo duas sessões de exercícios aeróbicos (60\%-75\% da frequência cardíaca máxima) e duas de exercícios resistidos (60\% 1RM). Entretanto, após três meses de cessação do programa ocorreram perdas significativas desses ganhos, o que indica que pacientes com DAC devem realizar continuamente exercícios físicos, a fim de melhorar e/ou manter a função cardiovascular e força muscular e, consequentemente, seu estado de saúde.

A associação de atividades aeróbicas e resistidas realizadas na água em pacientes cardíacos de baixo risco melhorou valores antropométricos, força muscular, capacidade aeróbica e o limiar de lactato após quatro meses de treinamento. Esses efeitos positivos adquiridos tenderam a diminuir parcialmente ou totalmente com o destreinamento nos quatro meses seguintes e foram recuperados com a retomada do programa por mais quatro meses (12).

Graças à importância da continuidade do treinamento, visando à manutenção dos benefícios promovidos pelo treinamento resistido associado ao exercício aeróbico, sugerida pelos artigos supracitados, a aderência de pacientes cardiopatas ao longo de 72 meses de treinamento resistido não supervisionado foi avaliada por meio da aplicação de um questionário de análise retrospectiva. A desistência ocorreu principalmente nos seis primeiros meses de treinamento, e o principal motivo apontado pelo abandono foi a frustração quanto à expectativa irreal de grande perda de peso. Os pacientes que permaneceram em treinamento relataram que o apoio familiar, de amigos e de seu médico responsável foi fundamental para a aderência ao programa. A taxa de interrupção por lesões musculares foi de apenas 3\%. 0 autor relata que a taxa de evasão dos programas não supervisionados é similar aos programas de treinamento supervisionado e sugere que, para minimizar a desistência, devem ser consideradas adequadas a supervisão e as orientações ao praticante, a fim de diminuir suas dúvidas quanto ao treinamento (15).

Em um ensaio clínico com intervenção durante um ano, 92 mulheres, após serem submetidas à angioplastia ou que apresentaram infarto agudo do miocárdio, foram subdivididas em dois grupos e submetidas a dois tipos de intervenção: treinamento combinado (aeróbico e resistido) ou exclusivamente aeróbico. As voluntárias foram avaliadas em relação à qualidade de vida (SF-36), força máxima e consumo máximo de oxigênio $\left(\mathrm{VO}_{2 \text { pico }}\right)$ nos períodos: inicial, dois meses, seis meses e após um ano de seguimento. Ambos os grupos apresentaram aumento do $\mathrm{VO}_{2 \text { pico' }}$, da força muscular e da qualidade 
de vida ao longo de um ano de seguimento, sem diferenças entre os grupos, indicando que as mulheres se beneficiaram tanto do programa combinado quanto do programa exclusivamente aeróbico (16).

A disfunção endotelial parece ser particularmente relevante em pacientes com doença arterial crônica que apresentam isquemia aguda ou crônica, por relacioná-los a um prognóstico pouco favorável, sendo, por meio da prática do exercício físico, possível melhorar essa função. Contudo, pouco se sabe dos efeitos produzidos da aplicação de diferentes tipos de modalidade de exercício. Para isso, investigou-se a resposta dessa função em pacientes pós-IAM submetidos aleatoriamente ao treinamento físico exclusivamente resistido ou aeróbico, ou ao exercício combinado (aeróbico e resistido). Os resultados mostraram que todos os grupos de exercício foram seguros e eficazes na correção da disfunção endotelial nesses pacientes, o que promove variedade na prescrição do exercício e influencia positivamente na aderência em longo prazo ao treinamento físico (17).

A revisão de autoria de Vicent and Vicent (5), baseando-se também nas recomendações do ACSM publicadas em 2000, recomenda a inclusão do treinamento resistido de baixa intensidade a partir da fase II dos programas de reabilitação cardiovascular, nas primeiras duas a três semanas após evento coronariano agudo. Essa recomendação foi aplicada no estudo prospectivo, randomizado e controlado de Schimid JP et al. (18), o qual investigou em pacientes cardíacos isquêmicos, com fração de ejeção de no máximo $45 \%$, os efeitos da prática de 12 semanas de exercício resistido (40\%-60\% de 1-RM) combinado ao aeróbico (70\%-85\% de frequência cardíaca de pico) em comparação ao exercício aeróbico isolado (70\%-85\% de frequência cardíaca de pico) no remodelamento do ventrículo esquerdo na fase aguda após IAM. Concluiu-se que a combinação do exercício não produziu dilatação do ventrículo esquerdo quando comparado ao grupo que realizou exercício aeróbico isolado e em ambos os grupos ocorreram aumentos significantes nos valores de $\mathrm{VO}_{2 \text { pico }}$ e força muscular, sem aumentos desproporcionais na frequência cardíaca e pressão arterial e o duplo produto permaneceu abaixo dos valores correspondentes da atividade aeróbica.

Volaklis and Tokmakidis (19), com base na revisão da literatura, apontam que a aplicação do treinamento com exercício de força dinâmica, realizado de forma isolada ou em combinação com exercícios aeróbicos, é segura e induz adaptações histoquímicas, metabólicas e funcionais na musculatura esquelética, que contribuem para neutralizar o estado de atrofia muscular periférica e melhorar a força muscular, influenciando de forma positiva na qualidade de vida de pacientes com IC estável. Essa revisão recomenda que o treinamento com 8-10 repetições deve ser realizado lentamente, em torno de 60 segundos de execução, seguido por um período de recuperação adequado, na proporção de 1:2, com intensidade geralmente na faixa de 50\%-60\% $1 \mathrm{RM}$. E, particularmente, pacientes com menor tolerância ao esforço podem realizar os exercícios de resistência de forma segmentar, por meio de pequenos pesos livres $(0,5$ a $3 \mathrm{~kg})$ ou cordas elásticas.

Da mesma forma, a revisão de Benton (20), realizada entre os anos de 1996 a 2006, sugere a inclusão do treinamento resistido como atividade rotineira em programa de exercício terapêutico, visto que essa modalidade mostra-se segura e eficiente para pacientes com IC na promoção de ganho de força e capacidade funcional, bem como melhora do comportamento hemodinâmico. Para manutenção desses benefícios, o treinamento deve ser continuado ao longo da vida. Segundo o autor, o teste de 1RM tem sido consistentemente recomendado como técnica segura para a mensuração da força muscular nessa população.

Feiereisen et al. (21) avaliaram a fração de ejeção do VE, consumo de oxigênio de pico, carga de pico, volume do músculo da coxa, força do extensor do joelho, resistência e qualidade de vida de 60 pacientes portadores de insuficiência cardíaca, submetidos a 40 sessões de treinamento com duração de 45 minutos cada, três vezes por semana. Os voluntários foram divididos em quatro grupos que realizaram: treinamento exclusivamente resistido, treinamento aeróbico isolado, treinamento combinado (aeróbico e resistido) e um grupo controle sem atividade. Todos os parâmetros mensurados melhoraram de forma significativa nos três grupos de exercício, exceto para a força de extensores do joelho no grupo de exercício aeróbico. Dessa maneira, independentemente da modalidade o treinamento físico é eficiente no aumento da função cardíaca, na capacidade do exercício, na função muscular periférica e na qualidade de vida em pacientes com IC.

Para entender os efeitos da combinação entre treinamento aeróbico e resistido foram selecionados 285 
pacientes com IC, entre homens e mulheres, os quais realizaram exercício de força muscular (2-3 vezes por semana com 3 a 5 séries e 20 repetições para MMSS e MMII com intensidades diferenciadas definidas a partir do $\mathrm{VO}_{2 \text { ico }}$ ) associado ao exercício de bicicleta e caminhada. Os autores observaram aumentos na fração de ejeção do ventrículo esquerdo e na força muscular, tanto nos homens quanto nas mulheres. Contudo, mudanças no pico de oxigênio foram verificadas apenas no grupo dos homens. Além disso, ambos os grupos aumentaram significantemente as suas distâncias. Os autores confirmaram a viabilidade de um programa de exercício combinado (aeróbico e resistido) e sugeriram a realização de atividade não supervisionada domiciliar, a fim de manutenção dos benefícios (22).

Outro estudo de Beckers et al. (23) teve como objetivo comparar os efeitos promovidos pelo treinamento resistido combinado ao aeróbico (TC: 6 a 8 tipos de exercício, 50\%-60\% 1RM, 1 a 2 séries, 10 a 15 repetições) e pelo treinamento aeróbico exclusivo (TA: $90 \% \mathrm{FC}_{\text {pico }}$ ) em 58 pacientes com IC, classe funcional II e III. Após seis meses de intervenção, o TC promoveu melhorias mais acentuadas para capacidade funcional submáxima, força muscular e qualidade de vidas desses pacientes, quando comparados aos efeitos promovidos pelo TA. Além disso, os autores relataram a ausência de efeitos desfavoráveis sobre os parâmetros do remodelamento do ventrículo esquerdo, apontando esse resultado como animador e convincente para implementação desse tipo de treinamento.

\section{Conclusão}

Os artigos revisados sugerem que o treinamento resistido é uma modalidade de exercício importante para a população cardiopata, pois, independente da variada metodologia utilizada na sua prescrição, mostrou-se eficiente para aumentar a força muscular de membros superiores e inferiores, uma das principais habilidades físicas que contribuem para melhora da capacidade funcional e da qualidade de vida desses pacientes.

Além disso, essa modalidade também pode ser considerada segura a essa população, uma vez que todos os artigos selecionados afirmam boa tolerância de seus voluntários ao longo do período de treinamento e ausência de eventos ou complicações cardiovasculares. Entretanto, deve ser destacada a importância de avaliações prévias específicas e in- dividuais, sobre as quais foram baseadas as prescrições dos componentes do treinamento.

Em relação à prescrição dos componentes específicos do treinamento resistido, as fontes selecionadas para essa revisão convergem quanto à aplicação do teste de 1RM como modo de avaliação para força muscular; todavia, em relação aos outros componentes da prescrição mostram-se bastante diversificadas. No entanto, pode-se observar maior frequência de recomendação do treinamento resistido associado ao treinamento aeróbico e às seguintes características: 6 a 10 tipos diferentes de exercício envolvendo a maioria dos grupos musculares de membros superiores e inferiores, 3 séries de exercício, 10 a 15 repetições, intensidade de carga entre $60 \%$ a $75 \%$ de 1RM com sobrecarga a partir do aumento do número de repetições ou da carga do componente estático, execução das repetições de forma ritmadas e controladas em até dois segundos para cada fase (concêntrica e excêntrica), proporção de 2:1 entre o período de execução e recuperação entre números de séries e repetições e, no mínimo, frequência de duas vezes na semana e quatro semanas de treinamento.

\section{Referências}

1. Taranto G, editor. Modificações na prescrição de exercício para pacientes cardíacos In: Diretrizes do ACSM para os testes de esforços e sua prescrição, American College of Sports Medicine. 7a ed. Rio de Janeiro: Guanabara Koogan; 2007.

2. Williams MA, Haskell WL, Ades PA, Amsterdam EA, Bittner V. Franklin BA, et al. Resistance exercise in individuals with and without cardiovascular disease: 2007 Update - A scientific statement from the American Heart Association council on clinical cardiology and council on nutrition, physical activity, and metabolism. Circulation. 2007;116(5):572-84.

3. Moraes RS, editores. Diretriz de Reabilitação Cardíaca. Arq Bras Cardiol. 2005;84(5):431-40.

4. Marzolini S, Oh P, Thomas SG, Goodman JM. Aerobic and resistance training in coronary disease: single versus multiple Sets Med Sci Sports Exerc. 2008;40(9):1557-64.

5. Vincent KR, Vincent HK. Resistance training for individuals with cardiovascular disease. J Cardiopulm Rehabil. 2006;26(4):207-16. 
6. Adams J, Cline M, Reed M, Masters A, Ehlke K, Hartman J. Importance of resistance training for patients after a cardiac event. Proc (Bayl Univ Med Cent). 2006;19(3):246-8.

7. Ghroubi S, Chaaria M, Elleucha H, Massmoudib K, Abdenadherc M, Trabelssid I, et al. The isokinetic assessment of peripheral muscle function in patients with coronary artery disease: correlations with cardiorespiratory capacity. Ann Readapt Med Phys. 2007;50(5):295-301

8. Okada Y, Toth MJ, VanBuren P. Skeletal muscle contractile protein function is preserved in human heart failure. J Appl Physiol. 2008;104(4):952-7.

9. Baum K, Hildebrandt U, Edel K, Bertram R, Hahmann $\mathrm{H}$, Bremer FJ, et al. Comparison of skeletal muscle strength between cardiac patients and age-matched healthy controls. Int J Med Sci. 2009;6(4):184-91.

10. Conraads VMA, Hoymans VY, Vrints CJ. Heart failure and cachexia: insights offered from molecular biology. Front Biosci. 2008;13:325-35.

11. Gunn E, Smith KM, McKelvie RS, Arthur H M. Exercise and the heart failure patient: aerobic vs strength training - is there a need for both? Prog Cardiovasc Nurs. 2006;21(3):146-50.

12. Tokmakidis SP, Spassis AT, Volaklis KA.Training, detraining and retraining effects after a water-based exercise program in patients with coronary artery disease. Cardiology. 2008;111(4):257-64.

13. Lamotte $M$, Niset $G$ and Borne $P$ van de. The effect of different intensity modalities of resistance training on beat-to-beat blood pressure in cardiac patients. Eur J Cardiovasc Prev Rehabil. 2005;12(1):12-7.

14. Volaklis KA, Douda HT, Kokkinos PF Tokmakidis SP. Physiological alterations to detraining following prolonged combined strength and aerobic training in cardiac patients. Eur J Cardiovasc Prev Rehabil. 2006;13(3):375-80.

15. Marzolini S, Mertens DJ, Oh PI, Plyley MJ. Selfreported compliance to home-based resistance training in cardiac patients. Eur J Cardiovasc Prev Rehabil. 2010;17(1):35-41.
16. Arthur HM, Gunn E, Thorpe KE, Ginis KM, Mataseje L, McCartney N, et al. Effect of aerobic vs combined aerobic-strength training on 1-year, post-cardiac rehabilitation outco mes in women after a cardiac event. J Rehabil Med. 2007;39(9):730-5.

17. Vona M, Codeluppi GM, Iannino T, Ferrari E, Bogousslavsky J, Segesser LK. Effects of different types of exercise training followed by detraining on endothelium-dependent dilation in patients with recent myocardial infarction. Circulation. 2009;119(12):1601-8.

18. Schmid J, Anderegg $M$, Romanens $M$, Morger $C$, Noveanu M, Hellige G, et al. Combined endurance/ resistance training early on, after a first myocardial infarction, does not induce negative left ventricular remodeling. Eur J Cardiovasc Prev Rehabil. 2008;15(3):341-6.

19. Volasklis AK, Savvas PT. Resistance exercise training in patients with heart failure. Sports Med. 2005;35(12):1085-103.

20. Benton MJ. Safety and efficacy of resistance training in patients with chronic heart failure: research-based evidence. Prog Cardiovasc Nurs. 2005;20(1):17-23.

21. Feiereisen P, Delagardelle C, Vaillant M, Lasar Y, Beissel J. Is strength training the more efficient training modality in chronic heart failure? Med Sci Sports Exerc. 2007;39(11):1910-7.

22. Miche E, Roelleke E, Wirtz U, Zoller B, Tietz M, Huerst $\mathrm{M}$, et al. Combined endurance and muscle strength training in female and male patients with chronic heart failure. Clin Res Cardiol. 2008;97(9):615-22.

23. Beckers PJ, Denollet J, Possemiers NM, Wuyts FL, Vrints CJ, Conraads VM. Combined endurance-resistance training vs. endurance training in patients with chronic heart failure: a prospective randomized study. Eur Heart J. 2008;29(15):1858-66.

Recebido: 05/04/2011 Received: 04/05/2011

Aprovado: 19/07/2011

Approved: 07/19/2011 